\title{
Asymmetric cell division of stem cells in the lung and other systems
}

\author{
Mohamed Berika ${ }^{1+}$, Marwa E. Elgayyar ${ }^{2+}$ and Ahmed H. K. El-Hashash ${ }^{3 *}$ \\ ${ }^{1}$ Rehabilitation Science Department, College of Applied Medical Sciences, King Saud University, KSA and Anatomy Department, Faculty of Medicine, Mansoura \\ University, Mansoura, Egypt \\ ${ }^{2}$ Department of Pediatric and Neonatology, Benha Children Hospital, Benha City, Egypt \\ ${ }^{3}$ Developmental Biology, Stem Cells and Regenerative Medicine Program, Keck School of Medicine and Ostrow School of Dentistry, Children's Hospital Los \\ Angeles, University of Southern California, Los Angeles, USA
}

Edited by:

Frederic Michon, University of Helsinki, Finland

\section{Reviewed by:}

Govindan Dayanithi, Institute of

Experimental Medicine of the

Academy of Sciences of the Czech

Republic, Czech Republic

Matthew Kirkham, Karolinska

Institutet, Sweden

*Correspondence:

Ahmed H. K. El-Hashash,

Developmental Biology, Stem Cells

and Regenerative Medicine

Program, Keck School of Medicine

and Ostrow School of Dentistry,

Children's Hospital Los Angeles,

University of Southern California,

4661 Sunset Boulevard MS 35, Los

Angeles, CA 90027, USA

e-mail: aelhashash@chla.usc.edu

${ }^{\dagger}$ These authors have contributed

equally to this work.
New insights have been added to identification, behavior and cellular properties of embryonic and tissue-specific stem cells over the last few years. The modes of stem cell division, asymmetric vs. symmetric, are tightly regulated during development and regeneration. The proper choice of a stem cell to divide asymmetrically or symmetrically has great consequences for development and disease because inappropriate asymmetric division disrupts organ morphogenesis, whereas uncontrolled symmetric division induces tumorigenesis. Therefore, understanding the behavior of lung stem cells could identify innovative solutions for restoring normal morphogenesis and/or regeneration of different organs. In this concise review, we describe recent studies in our laboratory about the mode of division of lung epithelial stem cells. We also compare asymmetric cell division (ACD) in the lung stem cells with other tissues in different organisms.

\section{Keywords: stem cell, behavior, symmetric, asymmetric, cell division}

\section{INTRODUCTION}

There are two types of cell division in different organisms: symmetric and asymmetric. The major purpose of symmetric divisions is proliferation, and it therefore leads to expansion of cell populations. The symmetric division produces two identical daughter cells that acquire the same developmental fate; while the asymmetric division. On the contrary, asymmetric cell division (ACD) is a property of stem cells that gives rise to two daughter cells with different developmental fates: one daughter will differentiate along a specific lineage, whereas the other cell has the potential to renew stem cell identity and continue to divide in an asymmetric manner (Figure 1). The ability of cells to divide asymmetrically to produce two different cell types provides the cellular diversity found in every multicellular organism.

The mode of stem cell division is critical for their maintenance and expansion. Stem cells may undergo both symmetric and ACD s, instructed by diverse molecular, cellular, and environmental cues at discrete developmental stages. To distinguish these, one can look at differences in spindle orientation, or differential inheritance of cytoplasmic or membrane-bound proteins such as the cell fate determinant Numb and atypical protein kinase C

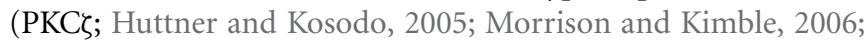
El-Hashash and Warburton, 2011, 2012; El-Hashash et al., 2011). Cells divide asymmetrically in response to extrinsic or intrinsic fate determinants: extrinsically, daughter cells placed in different microenvironments adopt different fates; intrinsically, cytoplasmic cell fate determinants (e.g., Numb) are asymmetrically localized within a cell and segregate differentially into daughters that adopt different fates (reviewed by Yamashita, 2009).

The mode of cell division: symmetric vs. ACDs can support stem cell self-renewal, and is critical to maintain the proper balance between self-renewal and differentiation of stem cells (Figure 1). For instance, symmetric cell division enables stem cells to generate two daughter cells, each with properties that are indistinguishable from the mother cell, which is necessary for expanding stem cell reservoirs if these two daughters acquire stem cell fate. In addition, symmetric cell division can produce two daughters that acquire a differentiation cell fate, and therefore have less potency than the mother stem cell (Figure 1). While this may lead to rapid production of tissue "effector" cells, it can also result in potential depletion of the stem cell pool (Molofsky et al., 2004; Yamashita et al., 2010). ACD, on the other hand, is essential for balancing self-renewal and differentiation as well as correct spatial and temporal specification of cell lineages during development. In contrast to symmetric division, $\mathrm{ACD}$ gives rise to two daughter cells with distinct cell fates. While one daughter maintains stem cell properties, the other daughter produced by ACD loses stem cell properties and functions (Figure 1; Knoblich, 


\section{Symmetric division}

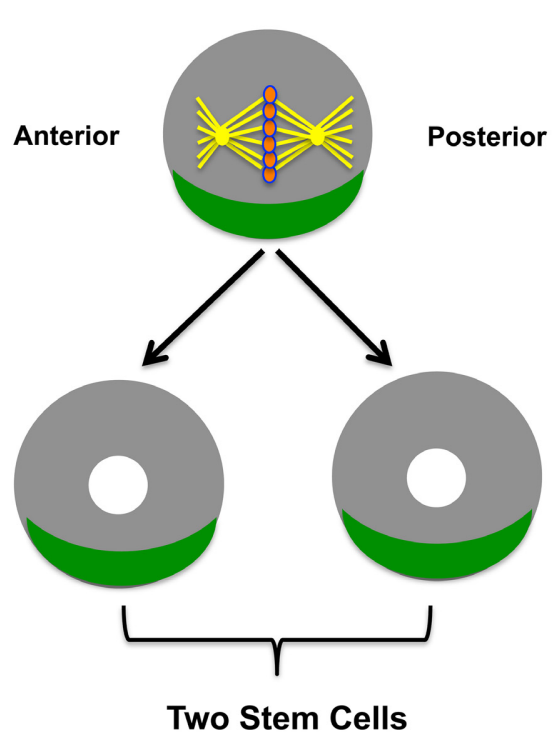

Asymmetric division

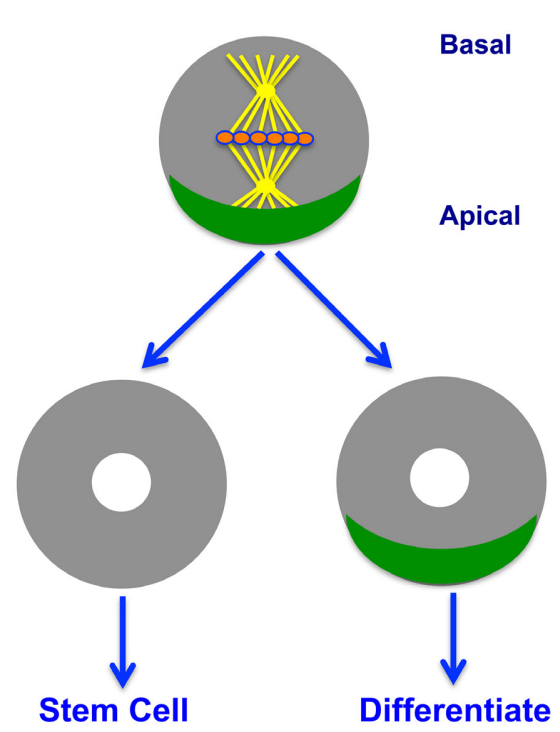

Cell-fate determinant protein (e.g. Numb)

FIGURE 1 | Symmetric vs. asymmetric cell division in epithelial cells. Schematic depiction of a polarized dividing cell show two modes of cell division. During symmetrical division, spindle orientation and determinant protein (e.g., Numb) localization are not coordinated. Determinants segregate equally, giving rise to two equal (stem) cells. During asymmetric division, spindle orientation and determinant protein (e.g., Numb) localization are coordinated, giving rise to a differentiating cell and a stem cell. Thus, the difference in Numb (green) expression levels between two daughter cells mediates asymmetric cell division, whereas lack of Numb inheritance by both daughters will allow them to execute the stem cell self-renewal program by maintaining Notch 1 activity and thus allowing symmetric cell division, as we reported in distal lung epithelial stem cells (El-Hashash and Warburton, 2012).
2001). Symmetric vs. ACD will be discussed in more detail in sections Asymmetry Stem Cell Divisions in Different Systems and Similarity and Significance of Asymmetric Stem Cell Division Between the Lung and Other Systems.

During development, cell divisions in the zygote produce various cell types. The ACD is the mechanism that provides the basis for many crucial developmental processes such as establishment of the body axis, cell fate determination, the maintenance of adult stem cell populations and generation of an adequate number of differentiating daughter cells. These differentiating daughter cells are vital to maintain tissue homeostasis and repair. Cell polarization is critical for asymmetric divisions. Disturbances or loss of cell polarity is often linked to enhanced stem cell self-renewal and tumorigenesis.

Asymmetric division is controlled by a combination of intrinsic and extrinsic mechanisms. Examples of intrinsic mechanisms are asymmetric localization of cell-cell junctions and/or intrinsic cell fate determinants and position within specific environment ("niche"). These are used to specify cell polarity and direct asymmetric divisions. Intrinsic mechanism involves the preferential segregation of cell fate determinants (e.g., Numb) into one of two daughter cells during mitosis (Figure 1). The successful segregation of determinants requires specialized machinery that mediates proper spindle orientation and coordinates other key events in this process. On the other hand, cell-cell communication, and thus establishment of different fates is reinforced through signaling from neighboring cells are examples of extrinsic mechanisms. For instance, interactions between daughter cells or between a daughter cell and other nearby cells in metazoans control the specification of daughter cell fate. However, multiple genes directly regulate ACDs in order to control the process of ACD itself and to determine the distinct cell fates of the two daughters.

\section{ASYMMETRIC CELL DIVISION IN MAMMALIAN LUNG EPITHELIAL STEM CELLS}

In mammals, control of epithelial stem cells is essential for proper development of the lung (Warburton, 2008; Warburton et al., 2010). Lung congenital deficiencies of stem and stem cells are lethal because they affect vital processes such as gas diffusion capacity, which occurs in lung hypoplasia and bronchopulmonary dysplasia (BPD; Warburton et al., 2000, 2008, 2010; Shi et al., 2009). Providing innovative solutions to restore normal lung morphogenesis and possibly regeneration of the gas diffusion surface needs understanding how to achieve a proper balance between self-renewal/proliferation and differentiation of lung-specific stem and stem cells. During development ACD is indeed critical for balancing self-renewal and differentiation as well as correct spatial and temporal specification of cell lineages in 
epithelia (see Knoblich, 2001; Yamashita et al., 2010, for detailed review).

Cell polarity is identified by asymmetry in the distribution of cellular constituents within a single cell. It is crucial for various cellular processes including cell specification and migration as well as asymmetric division. Cell polarity plays a fundamental role in helping to organize and integrate complex molecular signals so that cells can make decisions concerning fate, orientation, proliferation, differentiation, and interaction (Wodarz, 2002; Nelson, 2003). Recent studies in our laboratory on lung stem cells, for instance, have shown that lung stem cells are polarized and highly mitotic with characteristic perpendicular cell divisions (detailed below). Perturbation of the polarization of lung stem cells results in loss of balance between self-renewal and differentiation of lung stem cells in vivo and in culture (El-Hashash et al., 2011).

Understanding the behavior of lung epithelial stem and stem cells may help to identify innovative solutions to restore normal lung morphogenesis. The characterization of ACD and identifying novel factors and mechanisms regulating both ACD and behavior of lung epithelial stem cells, as key mechanisms that regulate the balance between stem cell self-renewal and differentiation in the lung, can help to identify novel targets which will prevent and rescue the fatal lung disease in infancy and childhood and for lung regeneration after injury. Furthermore, identification of the molecular programs regulating the balance between the proliferation and differentiation of endogenous lung-specific stem cells is critical for developing techniques that harness the ability of these cells to regenerate diseased and damaged lungs. Despite its importance, little is known about ACD in epithelial stem cells in the lung.

Undifferentiated epithelial stem cells undergo multiple division-linked cell fate decisions (symmetric and asymmetric) in the lung, which lead to an apparently homogeneous expansion of the stem cell population (Lu et al., 2008; Rawlins, 2008). Multipotent epithelial stem cells localize within the distal lung epithelial buds/airways during embryonic development (Rawlins and Hogan, 2006; Rawlins, 2008; Rawlins et al., 2009). Recently, studies from our laboratory have indicated that ACD likely mediates the balance between lung epithelial stem cell maintenance and differentiating cell populations at distal epithelial tips. The first evidence came from our laboratory that embryonic lung distal epithelial stem cells are is polarized and highly mitotic with characteristic perpendicular cell divisions. In different mammalian epithelial cells, perpendicular cell division is strictly correlated with ACD because they undergo asymmetric division by shifting the spindle orientation from parallel to perpendicular (Lechler and Fuchs, 2005). These findings are consistent with, mouse Inscuteable (mInsc), LGN (Gpsm2), and NuMA polarity proteins, which control spindle orientation, are asymmetrically localized in mitotic distal epithelial stem cells of embryonic lungs (El-Hashash and Warburton, 2011). Interfering with the function of these polarity proteins in lung epithelial cells in vitro randomizes spindle orientation and changes cell fate (El-Hashash et al., 2011).

ACD is mediated by preferential segregation of intrinsic cell fate determinants (CFDs) (e.g., Numb) into one of two sibling daughter cells in Drosophila and mammalian epithelial cells.
CFDs are asymmetrically localized in dividing cells and define the axis of polarity that will determine the orientation of the apical-basal cell division plane. This allows a rapid switch from proliferation, wherein two similar daughter cells are born, to diversification, wherein different-shaped daughter cells are generated (Betschinger and Knoblich, 2004). During interphase, Numb protein, a Notch signaling inhibitor, is expressed uniformly in the cytoplasm but is localized asymmetrically in dividing cells. Hence, Numb is segregated to only one daughter cell, enabling this cell to adopt a different fate from that of its sibling. The cell with low Numb levels maintains high Notch activity and thus has a stem cell fate whereas; the cell receiving high levels of Numb suppresses extrinsic Notch signaling and differentiates (Frise et al., 1996; Guo et al., 1996; Juven-Gershon et al., 1998; Yan et al., 2008). The cell fate determinant Numb in the embryonic lung is a key determinant of asymmetric or symmetric cell division, is highly expressed and asymmetrically distributed at the apical side of distal epithelial stem cells (El-Hashash and Warburton, 2011, 2012). Moreover, one of our recent findings is that Numb is segregated to one daughter cell in most mitotic cells (El-Hashash and Warburton, 2011). Thus, the more perpendicular/ACD is, the more likely it is to segregate Numb preferentially to one daughter cell in mitotic lung epithelial stem cells, which strongly suggest ACD in distal epithelial stem cells of embryonic lungs (El-Hashash and Warburton, 2012). Knocking down Numb in MLE15 lung epithelial cells significantly increased the number of cells expressing the stem cell markers Sox9/Id2, supporting its function as a cell fate determinant in the lung (El-Hashash and Warburton, 2012).

Epithelial cells characteristically show apical-basal polarity in many organs. They also have a distinct shape, such that only a subtle deviation in cleavage plane from the normal orientation suffices to result in an asymmetric rather than a symmetric distribution of their apical plasma membrane and adjacent adherent junctions to the daughter cells (Nelson, 2003; Kosodo et al., 2004). Using immunostaining for E-cadherin, which is a component of the apico-lateral junctional complex and lateral epithelial cell plasma membrane (Woods et al., 1997), the plasma membrane of mitotic epithelial cells show the "cadherin hole" which appears as a relatively small, unstained segment of the cell surface (Kosodo et al., 2004; El-Hashash and Warburton, 2012). Symmetric vs. asymmetric distribution of the plasma membrane to daughter cells can thus be predicted from the orientation of the cleavage plane relative to the cadherin hole in the epithelium of different organs (Kosodo et al., 2004). In our laboratory a focus on cadherin hole analyses of the lung epithelium revealed that most distal epithelial stem cells in embryonic lungs divide asymmetrically; with their cleavage, planes are predicted to bypass the cadherin hole, resulting in asymmetric distribution of the cadherin hole to the daughter cells. This provides evidence for ACD in distal epithelial stem cells of embryonic lungs (El-Hashash and Warburton, 2012).

In addition, in our laboratory we have shown that Eyal protein phosphatase regulates cell polarity, spindle orientation and the localization of the cell fate determinant Numb, which functions as an inhibitor of Notch signaling. Thus, Eyal promotes both perpendicular division as well as Numb asymmetric segregation 
to one daughter in mitotic distal lung epithelium, probably by regulating aPKC $\zeta$ phosphorylation levels (El-Hashash et al., 2011). In addition, epithelial cell polarity and mitotic spindle orientation are defective after interfering with Eyal function in vivo or in vitro (El-Hashash et al., 2011). Furthermore, we have shown that in $\mathrm{Eyal}^{-/-}$lungs, perpendicular division is not maintained and Numb is segregated to both daughter cells in mitotic epithelial cells, leading to inactivation of Notch signaling. Moreover, they showed that genetic activation of Notch signaling, which promotes stem cell identity at the expense of differentiated cell phenotypes, could rescue the Eya1 ${ }^{-/-}$lung phenotype, which is characterized by increased epithelial differentiation but reduced branching and loss of epithelial stem cells.

In our laboratory, we have indicated that Eyal protein phosphatase controls the balance between self-renewal and differentiation of distal lung epithelial stem cells by regulating ACD, which is critical for the long-term maintenance of tissue self-renewal during development and in diseases. For instance, congenital lung hypoplasia and bronchopulmonary dysplasia (BPD), wherein a significant deficiency of stem cells probably occurs, are common features of human prematurity and/or lung injury and are thus major public health problems in human infancy. Proper balance between self-renewal and differentiation of lung-specific stem cells, which is mediated by ACD, is absolutely required for normal lung morphogenesis and regeneration. In order to generate a sufficiently large gas diffusion surface to sustain life, regulated outgrowth and branching of the epithelial tubes is essential. Defective differentiation and postnatal respiratory distress are direct results of developmental defects in this smooth progression (Warburton et al., 2008, 2010).

To summarize, our laboratory provides several lines of evidence suggesting that ACD s are common in embryonic distal lung epithelial stem cell populations. For instance, the cleavage plane orientations are predicted to bypass the cadherin hole, resulting in asymmetric distribution of the cadherin hole to the daughter cells in most distal epithelial stem cells (El-Hashash and Warburton, 2012). In addition, our report that most of the distal epithelial cells have apically localized Par, LGN, NuMA, and mInsc polarity proteins, with mitotic spindles aligned perpendicular to the basement membrane and a characteristic asymmetric segregation/inheritance of Numb (El-Hashash and Warburton, 2011), provides further evidence that they are dividing asymmetrically. There is a strict correlation between ACD and the apical localization of polarity proteins Par/LGN/NuMA/mInsc, which control spindle orientation in mammalian epithelial mitotic cells (Lechler and Fuchs, 2005), perpendicular alignment of mitotic spindles, and asymmetric Numb segregation in different Drosophila and mammalian epithelial cell types (Cayouette and Raff, 2002, 2003; Haydar et al., 2003; Noctor et al., 2004; Lechler and Fuchs, 2005). Further investigations are needed to determine asymmetric vs. symmetric division in embryonic lung distal epithelial stem cells.

\section{ASYMMETRY STEM CELL DIVISIONS IN DIFFERENT SYSTEMS}

ACD has been reported in different tissue types in the animal kingdom. Factors and molecular mechanisms that act to specify cell fate and orient mitotic spindles during ACD are still not fully understood. Activation of the Notch signaling pathway and/or asymmetric segregation of the Notch inhibitor Numb are common mechanisms of ACD across a number of stem cell systems. In this section, examples of ACD that are dependent on Notch signaling activity and occur in non-mammalian or mammalian systems will be reviewed.

\section{INTESTINAL STEM CELLS (ISCS) OF DROSOPHILA}

ISC is a well-studied model system for ACD. It is demonstrated that ISCs act to maintain the intestinal epithelium. ISCs reside within clusters of 2-3 basally located diploid cells, which are interspersed between polyploid enterocytes along the intestinal basement membrane. In addition, they generate the hormone-producing enteroendocrine cells and polyploid enterocytes (Micchelli and Perrimon, 2006; Ohlstein and Spradling, 2006).

Ohlstein and Spradling (2007) have shown that the Notch signaling activity is critical for mediating asymmetric division of ISCs in the midgut of adult flies. They also demonstrated that Notch target genes in the daughter enteroblasts were activated by ISCs signal through Delta. They noticed that Notch signaling is activated in the daughter enteroblast exclusively. In addition, only the ISC in a direct contact with the basement membrane stained positive for the Notch ligand Delta. Furthermore, Notch positivity was detected in all cells in the stem cell containing clusters (Ohlstein and Spradling, 2007). The molecular mechanism that facilitates the asymmetric division by blocking of Notch signaling activity within the ISC is not investigated thoroughly. However, the analysis of mitotic spindle orientation in dividing ISCs by many studies demonstrated that these stem cells divide non-randomly, so that the daughter ISC that remains in contact with the basement membrane remains an ISC, whereas the daughter cell that is displaced away undergo differentiation to form an enteroblast (Toledano and Jones, 2009). Orientations of the mitotic spindle and the mechanism(s) controlling this process in ISCs have not been characterized.

\section{HEMATOPOIETIC STEM CELLS (HSCs)}

Several studies suggest the importance of Notch signaling in regulating the fate of HSCs by blocking differentiation exactly as in Drosophila ISCs (Duncan et al., 2005; Wu et al., 2007). These studies showed that a Notch-responsive GFP reporter in transgenic mice could be used to enrich for hematopoietic stem cells. This transgenic Notch reporter strain, shows $\mathrm{GFP}^{+}$ cells contained approximately 40-60\% HSCs, whereas in differentiating precursors expression of GFP is significantly reduced (Duncan et al., 2005; Wu et al., 2007). In addition, this transgenic Notch reporter strain and real time imaging in order to visualize hematopoietic precursor cell divisions growing in culture. Furthermore, Duncan et al. (2005) and Wu et al. (2007) highlighted the importance of both extrinsic cues and intracellular factors in the control of hematopoietic precursor cell divisions. They used these research tools to show that different types of oncogenic chromosomal translocations such as BCR-ABL can affect on either the pattern of cell divisions or cell proliferation and survival of hematopoietic stem cells. However, further 
investigation is needed to understand the molecular mechanisms involved in the regulation of ACD by these factors. Important questions are still waiting for answers such as are the niche and the surrounding different cell types, including vascular endothelial cells, osteoblasts and stromal reticular cells also influence the orientation pattern of mitotic spindles and ACDs of HSCs? Furthermore, what is the relative importance of the ability of hematopoietic stem cells to divide asymmetrically to the blood homeostasis?

\section{MUSCLE STEM CELLS}

Adjacent to the mature myofibers, satellite cells reside beneath the basement membrane and are effectively acting as muscle stem cells. Satellite cells are normally quiescent but can be induced to enter the cell cycle upon injury. They are essential to maintain production of myoblasts during postnatal development and during muscle repair after injury. Different daughter cells in dividing muscle-lineage cells during muscle growth and regeneration show asymmetric segregation of older (immortal) and younger DNA strands (Cairns, 1975; Shinin et al., 2006; Conboy et al., 2007). Other studies in culture and in vivo have shown induced expression of several differentiation genes and differential localization of cell fate proteins; including Numb, within daughter cells. Those studies provide evidence that muscle stem cells can undergo ACDs (Conboy and Rando, 2002; Shinin et al., 2006; Kuang et al., 2007). The relative importance of ACD in muscle regeneration needs further investigation. In addition, the molecular mechanisms that control muscle satellite cell division in response to various environmental cues and factors involved in the determination of daughter cell fates yet have to be established.

\section{EPIDERMAL STEM CELLS IN MAMMALS}

The skin is the largest organ of the body. It provides a protective barrier against the outside world. Damage of that barrier is potentially lethal and must be repaired rapidly and efficiently. The cells of the basal cell layer of the epidermis proliferate periodically to replicate themselves and to produce the supra-basal layers, which move outward and eventually die. The molecular factors responsible for ACDs are conserved throughout evolution. Several studies on skin cells growing in culture and on mouse embryos in vivo have shown evidences of both symmetric and ACD in epidermal stem cells in mammals. In addition, there is evidence of ACDs within the basal layer of the esophageal epithelium (Seery and Watt, 2000). Furthermore, several studies have shown that stem cells in the basal layer of the epidermis replicate symmetrically to produce more stem cells and asymmetrically, to generate the stratified epithelium of the epidermis (Smart, 1970; Lechler and Fuchs, 2005). Using ACD, the epidermal stem cells produce two cells, one is a proliferative cell which remains in the basal layer in contact with the baso-lateral membrane and another cell which detaches from the basal membrane to become in the supra-basal' layer and displace apically toward the surface of the skin (Smart, 1970; Lechler and Fuchs, 2005). The supra-basal cells stop dividing and form the barrier layer of the skin by entering a differentiation program (Fuchs and Raghavan, 2002).

Lechler and Fuchs (2005), showed that the mitotic spindles are perpendicular to the basement membrane in asymmetrically dividing cells during stratification of the skin. In addition, they showed that basal stem cells use integrins and cadherins in order to achieve physical attachment to the underlying basement membrane. These adhesion molecules are important for spindle alignment. Furthermore, they reported a concentration of both growth factor receptors and integrins at the base of the cell that could influence stem cell stem cell maintenance and behavior. Lechler and Fuchs (2005) studied the perpendicular cell divisions and demonstrated that they act as a natural mechanism for the unequal partitioning of the signaling molecules derived from the basement membrane into the two daughters. In mammalian epithelial stem cells, the apical complexes of polarity proteins are formed opposite the basement membrane and are segregated into the supra-basal cell that to be ready for differentiation (Figure 2). In asymmetric divisions, the mitotic cells with perpendicular spindles have an apical crescent of cortical LGN, which is considered as the mammalian Pins ortholog. LGN, in turn, binds both mouse Inscuteable (mInsc) and Par3 at the apical cortex of the basal cells. Another polarity protein is the atypical PKC (aPKC $\zeta)$, which localizes to the apical cortex of the basal cells (Lechler and Fuchs, 2005; Figure 2). Furthermore, LGN binds the Mud ortholog NuMA, which tethers spindles at the poles (Du et al., 2001). Integrins and cadherins are essential for the apical localization of aPKC $\zeta$, the Par3-LGN-Inscuteable complex and NuMA-dynactin to align the spindle in basal stem cells (Lechler and Fuchs, 2005). However, the primary function of the apical polarity proteins in mammalian epithelial cells is the determination of mitotic spindle positioning and establishment of apico-basal polarity, rather than the specification of stem cell fate (see Macara, 2004a,b; Lechler and Fuchs, 2005; Suzuki and Ohno, 2006; Shin et al., 2007; Knoblich, 2010; Yamashita et al., 2010 for detailed review).

Another important factor of ACDs in mammalian epidermal stem cells is the transcription factor p63, which stimulates epidermal proliferation (Mills et al., 1999; Yang et al., 1999). Basal cells only divide symmetrically in the absence of p63. This suggested

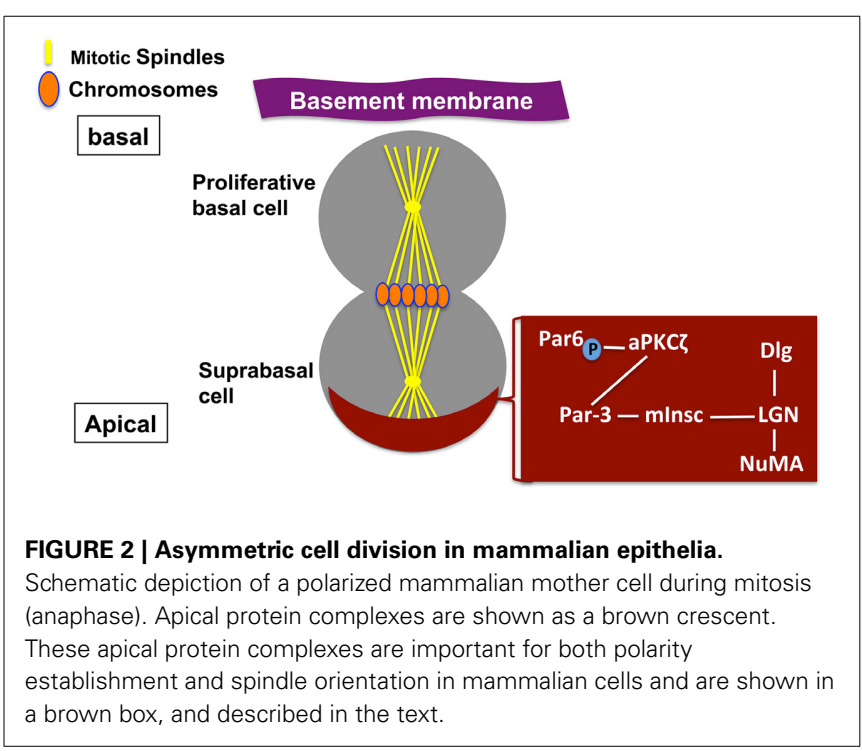


that p63 is required for stratification (Lechler and Fuchs, 2005; Senoo et al., 2007).

Similar to Drosophila neuroblasts, the asymmetric activation of the Notch pathway mechanism was used in order to ensure an asymmetric outcome of mammalian epithelial stem cell divisions. In addition, it was found that supra-basal cells utilize the Notch intracellular domain (NICD) to promote differentiation (Blanpain et al., 2006). Furthermore, Smith et al. (2007) have shown that Notch signaling is inhibited by the cell fate determinant Numb that localizes primarily to the baso-lateral cortex as a result of aPKC $\zeta$-mediated phosphorylation. This results in its exclusion from the apical pole in cultured mammalian epithelial cells. However, other molecular mechanisms and specific cell fate determinants that regulate ACDs, cell fate and behavior of mammalian epidermal stem cell during developmental and adult stages still need more investigation.

\section{MAMMALIAN NEURAL STEM CELLS}

Many authors have demonstrated that symmetric and ACDs occur at different developmental stages of neural stem cells in mammals. ACDs occur in the neuroepithelium of the vertebrate retina and ventricular zone of the cerebral cortex in mammals (Gonczy, 2008; Neumüller and Knoblich, 2009; Yamashita et al., 2010). Interestingly, during early developmental stages symmetric cell divisions also occur and are primarily reported probably to increase the neural stem cell population, while ACDs took place later on in order to generate differentiating neurons.

In the vertebrate nervous system, spindle orientation is regulated by several factors such as G $\alpha$-binding protein LGN, mouse Inscuteable (mInsc) and other factors have conserved roles similar to other systems. Furthermore, Notch signaling components are influence cell fate decisions in this system in vertebrate nervous system (Chenn and McConnell, 1995; Zhong et al., 1996, 1997; Petersen et al., 2004). Other studies suggest that notch signaling is inhibited by the cell fate determinant Numb. In addition, Numblike may influence cell fate by mechanisms other than inhibition of Notch signaling activity (Rasin et al., 2007; Zhou et al., 2007).

Knoblich $(2001,2010)$ has reviewed the role of the cell fate determinant Numb as a key determinant of asymmetric vs. symmetric cell division. Both ACD and Numb expression as well as function during development have been comparatively well studied in the mammalian nervous system and Drosophila (Betschinger and Knoblich, 2004), but they are not characterized in the lung.

During the development of vertebrate nervous system the correlation between mitotic spindle orientation and cell fate determination is not yet clear. In many other systems the spindle positioning is an indicator of whether cell divides symmetrically or asymmetrically (Sanada and Tsai, 2005; Zigman et al., 2005; Morin et al., 2007; Konno et al., 2008). For example, disruption of the activity of G $\alpha$-binding protein LGN, a key regulator of mitotic spindle orientation, in the spinal cord neuroepithelium leads to randomization of spindle orientation without disrupting daughter cell fate (Morin et al., 2007). On the other hand, reduction of another essential regulator of spindle orientation, mouse Inscuteable (mInsc) in the retina leads to disruption of mitotic spindle orientation and an increase in stem cells and neuronal defects (Zigman et al., 2005; see Figure 2).

Furthermore, a recent study by Forostyak and colleagues (Forostyak et al., 2013) has demonstrated the importance of $\mathrm{Ca}^{2+}$, its homeostasis and signaling potential in human embryonic stem cells (hESCs) differentiated to a neuronal phenotype. In this study, they have identified the molecular cascades of $\left[\mathrm{Ca}^{2+}\right]_{i}$ homeostasis and $\mathrm{Ca}^{2+}$ signaling in correlation with hESC differentiation into a neuronal phenotype. They have also analyzed changes in cytoplasmic $\mathrm{Ca}^{2+}$ concentration $\left(\left[\mathrm{Ca}^{2+}\right]_{i}\right)$ evoked by high $\mathrm{K}^{+}$, adenosine $-5^{\prime}$ triphosphate (ATP), glutamate, $\gamma$ aminobutyric acid (GABA), and caffeine in correlation with the expression of various neuronal markers in different passages (P6 through P10) during the course of hESC differentiation. This study has shown that only differentiated neural precursors (NPs) from $\mathrm{P} 7$ exhibited significant and specific $\left[\mathrm{Ca}^{2+}\right]_{i}$ responses to various stimuli. Thus, almost $31 \%$ of neuronal-like P7 NPs exhibited spontaneous $\left[\mathrm{Ca}^{2+}\right]_{i}$ oscillations. In addition, P7 NPs express L- and P/Q-type $\mathrm{Ca}^{2+}$ channels, $\mathrm{P} 2 \mathrm{X}_{2}, \mathrm{P}_{2} \mathrm{X}_{3}, \mathrm{P} 2 \mathrm{X}_{7}$, and $\mathrm{P} 2 \mathrm{Y}$ purinoreceptors, glutamate receptors, and ryanodine (RyR1 and RyR3) receptors (Forostyak et al., 2013). Moreover, they have provided evidences that the ATP- and glutamate-induced $\left[\mathrm{Ca}^{2+}\right]_{i}$ responses were concentration-dependent, and that responses to ATP were observed in the presence or in the absence of extracellular $\mathrm{Ca}^{2+}$. These data by Forostyak et al. (2013) strongly suggest that with time in culture, hESCs cells attain a transient period of operative $\mathrm{Ca}^{2+}$ signaling that is predictive of their ability to act as stem elements.

\section{SIMILARITY AND SIGNIFICANCE OF ASYMMETRIC STEM CELL DIVISION BETWEEN THE LUNG AND OTHER SYSTEMS}

Studies in our laboratory have shown a similarity in the mode of stem cell division between the lung and other systems. For instance, similar to stem cells of different tissues (Lechler and Fuchs, 2005; Yamashita et al., 2010), lung distal epithelial stem cells are polarized with perpendicular rather than parallel divisions (El-Hashash and Warburton, 2011). In addition, we found that asymmetric segregation and inheritance of Numb may be a common mode of ACD control in lung stem cells (El-Hashash et al., 2011; El-Hashash and Warburton, 2012), similar to neural stem cells and satellite muscle cells (reviewed in Morrison and Kimble, 2006). Another aspect of similarity is the apical localization of polarity proteins Par/LGN/NuMA/mInsc, which control spindle orientation in mammalian epithelial mitotic cells (Lechler and Fuchs, 2005). We reported that most distal lung epithelial stem cells have apically localized Par, LGN, NuMA, and mInsc polarity proteins, with mitotic spindles aligned perpendicular to the basement membrane. Both polarized localization of Par, LGN, NuMA, and mInsc proteins, and perpendicular alignment of mitotic spindles are strictly correlated with ACD in Drosophila and mammalian epithelial cell types (Cayouette and Raff, 2002, 2003; Haydar et al., 2003; Noctor et al., 2004; Lechler and Fuchs, 2005).

ACD mediates the balance between stem cell self-renewal and differentiation in different systems (Yamashita et al., 2010). This ACD-mediated balance is critical for the long-term maintenance of tissue self-renewal during development and in diseases in 
different organs, including the lung. For example, bronchopulmonary dysplasia (BPD) and congenital lung hypoplasia, wherein a significant deficiency of stem cells probably occurs, are common features of human prematurity and/or lung injury and are thus major public health problems in human infancy. Therefore, the proper balance between self-renewal and differentiation of lungspecific stem cells that is mediated by ACD, is most likely required for normal lung development, repair and regeneration. Indeed, tightly controlled outgrowth and branching of the epithelial tubes in the lung generate a sufficiently large gas diffusion surface to sustain life. Developmental defects in this smooth progression may, therefore, lead to defective differentiation and postnatal respiratory distress (Warburton et al., 2008, 2010). Our data that show similarities of asymmetric stem cell division between the lung and other systems could identify innovative solutions to restoring normal lung morphogenesis.

\section{CONCLUDING REMARKS AND FUTURE DIRECTIONS}

Much insight into different mechanisms that are necessary to generate cellular diversity and maintain stem cells have been demonstrated by several recent studies that focused on ACDs across various species and in multiple stem cell systems. Studies using invertebrate model systems such as Drosophila have identified the importance of several extrinsic signals and intrinsic factors in stem cell division pattern and provided paradigms for how both these signals and factors act to specify asymmetric divisions. Many recent studies provide evidence that similar mechanisms are used in vertebrates. However, proper characterization of stem cells in vivo, advanced isolation of pure populations of stem cells, and improvements in real time imaging are still needed to facilitate studies that aim at the identification and determination of the mechanisms regulating ACDs in more complex mammalian stem cell systems, including humans.

Proper balance of the number of stem and stem cells is also essential during organ development, repair and regeneration. Many recent studies on the mechanisms regulating asymmetric stem cell divisions have shown the importance of the balance of the number of stem cells. The correct balance and tight control of the number of stem cells by asymmetric divisions are not only important during the establishment and maintenance of tissues, but also critical during tissue repair and regeneration, This is because an increase in the number of symmetric divisions may be required temporarily to increase the number of stem cells during tissue repair and regeneration. It is important to consider that several factors can act to hinder or even prevent stem cell from switching from symmetric back to asymmetric mode of cell divisions. For instance, chronic injury or inflammation of a tissue might compromise the ability of stem cells to respond appropriately to repair damaged tissues. It also may cause failure of stem cells to switch from symmetric to asymmetric mode of divisions. Failure of the proper regulation of tissue repair could eventually lead to the selection of stem cells that are resistant to normal growth control signals, which is a hallmark of cancer cells. Therefore, understanding signaling mechanisms that regulate ACD in all types of stem cells is critical for developing techniques that harness the ability of these cells to regenerate diseased and damaged organs. In addition, understanding these mechanisms will most likely help to design potent strategies to inhibit cancer initiation in different cell types and may also identify new targets for anti-cancer therapies. Moreover, identification of the molecular mechanisms and factors regulating the behavior of adult stem cells will be essential for both the expansion and maintenance of stem cells in culture, while maintaining their differentiation potential. This will also direct the differentiation of stem cells into different specialized cell types ready for use in regenerative medicine.

Our recent studies on the characterization of $\mathrm{ACD}$, and future studies on the identification of new factors and mechanisms that regulate $\mathrm{ACD}$ in lung epithelial stem cells, which is a key mechanism regulating the balance between lung stem cell self-renewal and differentiation, may help to identify novel targets for the prevention and rescue therapy of fatal lung disease in infancy and childhood and for lung regeneration after injury. In addition, future studies on the identification of molecular programs that control the balance between self-renewal and differentiation of endogenous lung-specific stem cells will be crucial for developing techniques that harness the ability of these cells to regenerate diseased and damaged lungs.

\section{ACKNOWLEDGMENTS}

This work was supported by the American Heart Association National Scientist Development [grant number 12SDG12120007), the California Institute for Regenerative Medicine [grant number TG2-01168], and the Pasadena Guild Endowment to Ahmed H. K. El-Hashash. Dr. Mohamed Berika is financially supported by The Research Center of the College of Applied Medical Sciences, King Saud University, KSA.

\section{REFERENCES}

Betschinger, J., and Knoblich, J. A. (2004). Dare to be different: asymmetric cell division in Drosophila, C. elegans and vertebrates. Curr. Biol. 14, R674-R685. doi: 10.1016/j.cub.2004.08.017

Blanpain, C., Lowry, W. E., Pasolli, H. A., and Fuchs, E. (2006). Canonical notch signaling functions as a commitment switch in the epidermal lineage. Genes Dev. 20, 3022-3035. doi: 10.1101/gad.1477606

Cairns, J. (1975). Mutation selection and the natural history of cancer. Nature 255, 197-200. doi: 10.1038/255197a0

Cayouette, M., and Raff, M. (2002). Asymmetric segregation of Numb: a mechanism for neural specification from Drosophila to mammals. Nat. Neurosci. 5, 1265-1269. doi: 10.1038/nn1202-1265

Cayouette, M., and Raff, M. (2003). The orientation of cell division influences cellfate choice in the develop- ing mammalian retina. Development 130, 2329-2339. doi: 10.1242/dev.00446

Chenn, A., and McConnell, S. K. (1995). Cleavage orientation and the asymmetric inheritance of Notch1 immunoreactivity in mammalian neurogenesis. Cell 82, 631-641. doi: 10.1016/0092-8674(95)90035-7

Conboy, I. M., and Rando, T. A. (2002). The regulation of Notch signaling controls satellite cell activation and cell fate determination in postnatal myogenesis. Dev. Cell 3, 397-409. doi: 10.1016/S1534-5807(02)00254-X

Conboy, M. J., Karasov, A. O., and Rando, T. A. (2007). High incidence of nonrandom template strand segregation and asymmetric fate determination in dividing stem cells and their progeny. PLoS Biol. 5:e102. doi: 10.1371/journal.pbio.0050102

Du, Q., Stukenberg, P. T., and Macara, I. G. (2001). A mammalian Partner of inscuteable binds NuMA and regulates mitotic spindle organization. Nat. Cell Biol. 3, 1069-1075. doi: 10.1038/ncb1201-1069

Duncan, A. W., Rattis, F. M., DiMascio, L. N., Congdon, K. L., Pazianos, G., Zhao, C., et al. (2005). Integration of Notch and Wnt signaling in hematopoietic stem cell maintenance. Nat. Immunol. 6, 314-322. doi: 10.1038/ni1164 
El-Hashash, A., Turcatel, G., Alam, D., Buckley, S., Bellusci, S., and Warburton, D. (2011). Eyalcontrols cell polarity, spindle orientation, cell fate and Notch signaling in distal embryonic lung epithelium. Development 138, 1395-1407. doi: 10.1242/dev.058479

El-Hashash, A., and Warburton, D. (2011). Cell polarity and spindle orientation in the distal epithelium of embryonic lung. Dev. Dyn. 240, 441-445. doi: 10.1002/dvdy.22551

El-Hashash, A., and Warburton, D. (2012). Numb expression and asymmetric versus symmetric cell division in embryonic distal lung epithelium. J. Histochem. Cytchem. 60, 675-682. doi: 10.1369/0022155412451582

Forostyak, O., Romanyuk, N., Verkhratsky, A., Sykova, E., and Dayanithi, G. (2013). Plasticity of calcium signaling cascades in human embryonic stem cell-derived neural precursors. Stem Cells Dev. 22, 1506-1521. doi: 10.1089/scd.2012.0624

Frise, E., Knoblich, J., Younger-Shepherd, S., Jan, L. Y., and Jan, Y. N. (1996). The Drosophila Numb protein inhibits signaling of the Notch receptor during cell-cell interaction in sensory organ lineage. Proc. Natl. Acad. Sci. U.S.A. 93, 11925-11932. doi: 10.1073/pnas.93.21.11925

Fuchs, E., and Raghavan, S. (2002). Getting under the skin of epidermal morphogenesis. Nat. Rev. Genet. 3, 199-209. doi: 10.1038/nrg758

Gonczy, P. (2008). Mechanisms of asymmetric cell division: flies and worms pave the way. Nat. Rev. Mol. Cell Biol. 9, 355-366. doi: 10.1038/nrm2388

Guo, M., Jan, L., and Jan, Y. N. (1996). Control of daughter cell fates during asymmetric division: interaction of Numb and Notch. Neuron 17, 27-41. doi: 10.1016/S0896-6273(00)80278-0

Haydar, T. F., Ang, E. Jr., and Rakic, P. (2003). Mitotic spindle rotation and mode of cell division in the developing telencephalon. Proc. Natl. Acad. Sci. U.S.A. 100, 2890-2895. doi: 10.1073/pnas.0437969100

Huttner, W. B., and Kosodo, Y. (2005). Symmetric versus asymmetric cell division during neurogenesis in the developing vertebrate central nervous system. Curr. Opin. Cell Biol. 17, 648-657. doi: 10.1016/j.ceb.2005.10.005

Juven-Gershon, T., Shifman, O., Unger, T., Elkeles, A., Haupt, Y., and Oren, M. (1998). The Mdm2 oncoprotein interacts with the cell fate regulator Numb. Mol. Cell. Biol. 18, 3974-3982.

Knoblich, J. A. (2001). Asymmetric cell division during animal development. Nat. Rev. Mol. Cell Biol. 2, 11-20. doi: 10.1038/35048085

Knoblich, J. A. (2010). Asymmetric cell division: recent developments and their implications for tumour biology. Nat. Rev. Mol. Cell Biol. 11, 849-860. doi: $10.1038 / \mathrm{nrm} 3010$

Konno, D., Shioi, G., Shitamukai, A., Mori, A., Kiyonari, H., Miyata, T., et al. (2008). Neuroepithelial progenitors undergo LGN-dependent planar divisions to maintain self-renewability during mammalian neurogenesis. Nat. Cell Biol. 10, 93-101. doi: 10.1038/ncb1673

Kosodo, Y., Röper, K., Haubensak, W., Marzesco, A. M., Corbeil, D., and Huttner, W. B. (2004). Asymmetric distribution of the apical plasma membrane during neurogenic divisions of mammalian neuroepithelial cells. EMBO J. 23, 2314-2324. doi: 10.1038/sj.emboj.7600223

Kuang, S., Kuroda, K., Le Grand, F., and Rudnicki, M. A. (2007). Asymmetric selfrenewal and commitment of satellite stem cells in muscle. Cell 129, 999-1010. doi: 10.1016/j.cell.2007.03.044

Lechler, T., and Fuchs, E. (2005). Asymmetric cell divisions promote stratification and differentiation of mammalian skin. Nature 437, 275-280. doi: 10.1038 /nature 03922

Lu, Y., Okubo, T., Rawlins, E., and Hogan, B. L. (2008). Epithelial progenitor cells of the embryonic lung and the role of microRNAs in their proliferation. Proc. Am. Thorac Soc. 5, 300-304. doi: 10.1513/pats.200710-162DR

Macara, I. G. (2004a). Par proteins: partners in polarization. Curr. Biol. 14, R160-R162. doi: 10.1016/j.cub.2004.01.048

Macara, I. G. (2004b). Parsing the polarity code. Nat. Rev. Mol. Cell Biol. 5, 220-231. doi: $10.1038 / \mathrm{nrm} 1332$

Micchelli, C. A., and Perrimon, N. (2006). Evidence that stem cells reside in the adult Drosophila midgut epithelium. Nature 439, 475-479. doi: 10.1038 /nature 04371

Mills, A. A., Zheng, B., Wang, X. J., Vogel, H., Roop, D. R., and Bradley, A. (1999). p63 is a p53 homologue required for limb and epidermal morphogenesis. Nature 398, 708-713. doi: 10.1038/19531

Molofsky, A. V., Pardal, R., and Morrison, S. J. (2004). Diverse mechanisms regulate stem cell self-renewal. Curr. Opin. Cell Biol. 16, 700-707. doi: 10.1016/j.ceb.2004.09.004
Morin, X., Jaouen, F., and Durbec, P. (2007). Control of planar divisions by the G-protein regulator LGN maintains progenitors in the chick neuroepithelium. Nat. Neurosci. 10, 1440-1448. doi: 10.1038/nn1984

Morrison, S. J., and Kimble, J. (2006). Asymmetric and symmetric stem-cell divisions in development and cancer. Nature 441:7097. doi: 10.1038/nature 04956

Nelson, W. J. (2003). Epithelial cell polarity from the outside looking in. News Physiol. Sci. 18, 143-146. doi: 10.1152/nips.01435.2002

Neumüller, R. A., and Knoblich, J. A. (2009). Dividing cellular asymmetry: asymmetric cell division and its implications for stem cells and cancer. Genes Dev. 23, 2675-2699. doi: 10.1101/gad.1850809

Noctor, S. C., Martinez-Cerdeno, V., Ivic, L., and Kriegstein, A. R. (2004). Cortical neurons arise in symmetric and asymmetric division zones and migrate through specific phases. Nat. Neurosci. 7, 136-144. doi: 10.1038/ nn 1172

Ohlstein, B., and Spradling, A. (2006). The adult Drosophila posterior midgut is maintained by pluripotent stem cells. Nature 439, 470-474. doi: 10.1038 /nature04333

Ohlstein, B., and Spradling, A. (2007). Multipotent Drosophila intestinal stem cells specify daughter cell fates by differential notch signaling. Science 315, 988-992. doi: 10.1126/science.1136606

Petersen, P. H., Zou, K., Krauss, S., and Zhong, W. (2004). Continuing role for mouse Numb and Numbl in maintaining progenitor cells during cortical neurogenesis. Nat. Neurosci. 7, 803-811. doi: 10.1038/nn1289

Rasin, M. R., Gazula, V. R., Breunig, J. J., Kwan, K. Y., Johnson, M. B., Liu-Chen, S., et al. (2007). Numb and Numbl are required for maintenance of cadherinbased adhesion and polarity of neural progenitors. Nat. Neurosci. 10, 819-827. doi: $10.1038 / \mathrm{nn} 1924$

Rawlins, E. (2008). Lung epithelial progenitor cells: lessons from development. Proc. Am. Thorac. Soc. 5, 675-681. doi: 10.1513/pats.2008 01-006AW

Rawlins, E., Clark, C., Xue, Y., and Hogan, B. L. (2009). The Id2+ distal tip lung epithelium contains individual multipotent embryonic progenitor cells. Development 136, 3741-3745. doi: 10.1242/dev.037317

Rawlins, E., and Hogan, B. L. (2006). Epithelial stem cells of the lung: privileged few or opportunities for many? Development 133, 2455-2465. doi: 10.1242/dev.02407

Sanada, K., and Tsai, L. H. (2005). G protein betagamma subunits and AGS3 control spindle orientation and asymmetric cell fate of cerebral cortical progenitors. Cell 122, 119-131. doi: 10.1016/j.cell.2005.05.009

Seery, J. P., and Watt, F. M. (2000). Asymmetric stem-cell divisions define the architecture of human oesophageal epithelium. Curr. Biol. 10, 1447-1450. doi: 10.1016/S0960-9822(00)00803-4

Senoo, M., Pinto, F., Crum, C. P., and McKeon, F. (2007). p63 Is essential for the proliferative potential of stem cells in stratified epithelia. Cell 129, 523-536. doi: 10.1016/j.cell.2007.02.045

Shi, W., Xu, J., and Warburton, D. (2009). Development, repair and fibrosis: what is common and why it matters. Respirology 14, 656-665. doi: 10.1111/j.14401843.2009.01565.x

Shin, K., Wang, Q., and Margolis, B. (2007). PATJ regulates directional migration of mammalian epithelial cells. EMBO Rep. 8, 158-164. doi: 10.1038/sj.embor.7400890

Shinin, V., Gayraud-Morel, B., Gomes, D., and Tajbakhsh, S. (2006). Asymmetric division and cosegregation of template DNA strands in adult muscle satellite cells. Nat. Cell Biol. 8, 677-687. doi: 10.1038/ncb1425

Smart, I. H. (1970). Variation in the plane of cell cleavage during the process of stratification in the mouse epidermis. Br. J. Dermatol. 82, 276-282. doi: 10.1111/j.1365-2133.1970.tb12437.x

Smith, C. A., Lau, K. M., Rahmani, Z., Dho, S. E., Brothers, G., She, Y. M., et al. (2007). aPKC-mediated phosphorylation regulates asymmetric membrane localization of the cell fate determinant Numb. EMBO J. 26, 468-480. doi: 10.1038/sj.emboj.7601495

Suzuki, A., and Ohno, S. (2006). The PAR-aPKC system: lessons in polarity. J. Cell Sci. 119, 979-987. doi: 10.1242/jcs.02898

Toledano, H., and Jones, D. L. (2009). "Mechanisms regulating stem cell polarity and the specification of asymmetric divisions," in StemBook [Internet] (Cambridge, MA: Harvard Stem Cell Institute), 2008. Available onlie at: http:// www.ncbi.nlm.nih.gov/books/NBK27028/ 
Warburton, D. (2008). Developmental biology: order in the lung. Nature 453, 733-735. doi: 10.1038/453733a

Warburton, D., El-Hashash, A., Carraro, G., Tiozzo, C., Sala, F., Rogers, O., et al. (2010). Lung organogenesis. Curr. Top. Dev. Biol. 90, 73-158. doi: $10.1016 / 50070-2153(10) 90003-3$

Warburton, D., Perin, L., Defilippo, R., Bellusci, S., Shi, W., and Driscoll, B. (2008). Stem/progenitor cells in lung development, injury repair, and regeneration. Proc. Am. Thorac. Soc. 5, 703-706. doi: 10.1513/pats.2008 01-012AW

Warburton, D., Schwarz, M., Tefft, D., Flores-Delgado, G., Anderson, K. D., and Cardoso, W. V. (2000). The molecular basis of lung morphogenesis. Mech. Dev. 92, 55-81. doi: 10.1016/S0925-4773(99)00325-1

Wodarz, A. (2002). Establishing cell polarity in development. Nat. Cell Biol. 4, E39-E44. doi: 10.1038/ncb0202-e39

Woods, D. F., Wu, J.-W., and Bryant, P. J. (1997). Localization of proteins to the apico-lateral junctions of Drosophila epithelia. Dev. Genet. 20, 111-118. doi: 10.1002/(SICI) 1520-6408(1997)20:2<111::AID-DVG4>3.0.CO;2-A

Wu, M., Kwon, H. Y., Rattis, F., Blum, J., Zhao, C., Ashkenazi, R., et al. (2007). Imaging hematopoietic precursor division in real time. Cell Stem Cell. 1, 541-554. doi: 10.1016/j.stem.2007.08.009

Yamashita, Y. M. (2009). The centrosome and asymmetric cell division. Prion 3, 84-88. doi: 10.4161/pri.3.2.8821

Yamashita, Y. M., Yuan, H., Cheng, J., and Hunt, A. J. (2010). Polarity in stem cell division: asymmetric stem cell division in tissue homeostasis. Cold Spring Harb. Perspect. Biol. 2:a001313. doi: 10.1101/cshperspect.a001313

Yan, B., Omar, F. M., Das, K., Ng, W. H., Lim, C., Shiuan, K., et al. (2008). Characterization of Numb expression in astrocytomas. Neuropathology 28, 479-484. doi: 10.1111/j.1440-1789.2008.00907.x

Yang, A., Schweitzer, R., Sun, D., Kaghad, M., Walker, N., Bronson, R. T., et al. (1999). p63 is essential for regenerative proliferation in limb, craniofacial and epithelial development. Nature 398, 714-718. doi: 10.1038/ 19539
Zhong, W., Feder, J. N., Jiang, M. M., Jan, L. Y., and Jan, Y. N. (1996). Asymmetric localization of a mammalian numb homolog during mouse cortical neurogenesis. Neuron 17, 43-53. doi: 10.1016/S0896-6273(00)80279-2

Zhong, W., Jiang, M. M., Weinmaster, G., Jan, L. Y., and Jan, Y. N. (1997). Differential expression of mammalian Numb, Numblike and Notch1 suggests distinct roles during mouse cortical neurogenesis. Development 124, 1887-1897.

Zhou, Y., Atkins, J. B., Rompani, S. B., Bancescu, D. L., Petersen, P. H., Tang, H., et al. (2007). The mammalian Golgi regulates numb signaling in asymmetric cell division by releasing ACBD3 during mitosis. Cell 129, 163-178. doi: 10.1016/j.cell.2007.02.037

Zigman, M., Cayouette, M., Charalambous, C., Schleiffer, A., Hoeller, O., Dunican, D., et al. (2005). Mammalian inscuteable regulates spindle orientation and cell fate in the developing retina. Neuron 48, 539-545. doi: 10.1016/j.neuron.2005.09.030

Conflict of Interest Statement: The authors declare that the research was conducted in the absence of any commercial or financial relationships that could be construed as a potential conflict of interest.

Received: 25 April 2014; accepted: 14 July 2014; published online: 31 July 2014. Citation: Berika M, Elgayyar ME and El-Hashash AHK (2014) Asymmetric cell division of stem cells in the lung and other systems. Front. Cell Dev. Biol. 2:33. doi: 10.3389/ fcell.2014.00033

This article was submitted to Stem Cell Treatments, a section of the journal Frontiers in Cell and Developmental Biology.

Copyright () 2014 Berika, Elgayyar and El-Hashash. This is an open-access article distributed under the terms of the Creative Commons Attribution License (CC BY). The use, distribution or reproduction in other forums is permitted, provided the original author(s) or licensor are credited and that the original publication in this journal is cited, in accordance with accepted academic practice. No use, distribution or reproduction is permitted which does not comply with these terms. 